\title{
Autologous Fat Graft in the Reconstructed Breast: Fat Absorption Rate and Safety based on Sonographic Identification
}

\author{
Hong Youl Kim, Bok Ki Jung, Dae Hyun Lew, Dong Won Lee \\ Department of Plastic and Reconstructive Surgery, Institute for Human Tissue Restoration, Severance Hospital, Yonsei University College of \\ Medicine, Seoul, Korea
}

Background Autologous fat graft has become a useful technique for correction of acquired contour deformity in reconstructed breasts. However, there remains controversial regarding the efficacy and safety of the practice for reconstructive breast surgery.

Methods A retrospective review was performed on 102 patients who had secondary fat grafting after breast reconstruction. Fat harvest, refinement and injection were done by Coleman's technique. All patients were followed up postoperatively within 1 month and after 6 months including physical examination and ultrasonography. In 38 patients, the reabsorption rate was calculated by serial changes of thickness between skin and pectoral fascia in the ultrasonic finding. Locoregional recurrence rate was compared with control group of 449 patients who had breast reconstruction without fat graft in the same time period.

Results Average $49.3 \mathrm{~mL}$ fat was injected into each breast. The most common location of fat graft was upper pole, followed by axilla, lower and medial breasts. During 28.7 months of average follow-up period, $2.9 \%$ of total patients had symptoms of palpable mass on fat graft side and ultrasonography identified fat necrosis and cyst formation in $17.6 \%$ of the patients. Calculated fat reabsorption rate was 32.9\%. Locoregional recurrence was occurred in 1 patient $(0.9 \%)$ and the rate was not different significantly with control group (2\%).

Conclusions Although further studies are required to provide surgeons with definitive guidelines for the implementation of fat grafting, we propose autologous fat graft is an efficient and safe technique for secondary breast reconstruction.

Keywords Breast / Fat necrosis / Ultrasonography
Correspondence: Dong Won Lee Department of Plastic and Reconstructive Surgery, Yonsei University College of Medicine, 50 Yonsei-ro, Seodaemun-gu, Seoul 120-752, Korea

Tel: +82-2-2228-2215 Fax: +82-2-393-6947 E-mail:xyphoss@yuhs.ac
No potential conflict of interest relevant to this article was reported.

\section{INTRODUCTION}

Reconstructive techniques using flaps or implants to preserve the enveloping skin, and even the nipple areolar complex, have undergone vast improvements in recent years. Secondary refinements of the shape of reconstructed breasts have become rela- tively routine as patients and plastic surgeons desire further improvements, and many plastic surgeons consider the technique of autologous fat grafting for this purpose [1]. Although the American Society of Plastic and Reconstructive Surgeons' position paper in 1987 stated that fat grafting would compromise breast cancer detection and should therefore be prohibited [2], 
there is currently no evidence that fat grafting to breasts is less safe than any other form of breast surgery. Moreover, since the mid-2000s, several plastic surgeons reported good results using autologous fat grafting to correct contour deformities in the reconstructed breasts $[3,4]$.

However, controversy remains regarding the efficacy and safety of autologous fat grafts for reconstructive breast surgery. There are several potential drawbacks of autologous fat grafts in reconstructed breast. These include interference with the subsequent diagnosis of breast cancer by radiologic studies; unpredictable fat reabsorption rates, which may affect the efficacy; and a possible increased risk of neoplasia [5]. Furthermore, it has been suggested that growth factors derived from adipocytes may stimulate the formation of breast cancer [6]. It is important to reach a consensus about these concerns to determine the most appropriate use of fat graft in patients with prior breast cancer surgery.

The aim of this study was to identify the long-term efficacy and safety of autologous fat graft after mastectomy and reconstruction by implants or flaps in patients with breast cancer. We examined rate of such complications as fat necrosis and cyst formation noted on follow-up ultrasounds, the graft reabsorption rate, and the rate of cancer recurrence.

\section{METHODS}

\section{Patients}

The study included 102 patients with a history of breast cancer who underwent autologous fat graft for secondary revision breast surgery between January 2005 and August 2013. Their mean age was 46.3 years (range, 22-63 years). The type of original reconstruction surgery was transverse rectus abdominis myocutaneous free flap reconstruction $(\mathrm{n}=15)$, latissimus dorsi (LD) myocutaneous island flap ( $\mathrm{n}=41)$, or implant reconstruction $(n=46)$. For 90 patients, the reconstruction was immediate, being performed in conjunction with the general surgery team, whereas for 12 patients, the reconstruction was delayed. Fat graft was performed on the left breast in 53 patients and on the right side in 49 patients. The type of cancer was ductal carcinoma in situ $(n=42)$, stage I $(n=39)$, stage IIA $(n=13)$, stage IIB $(n=7)$, and stage IIIA $(n=1)$. The mean time interval between mastectomy and fat graft was 12.5 months (range, 3.262.2 months).

We estimated the data statistically with generalized estimating equations (GEE). Because a patient may have multiple fat grafting sites, we analyzed the correlation between fat grafting sites and operation methods with GEE, not chi-square test which is used more generally. As a result of the estimation, we found that there was no statistic correlation $(\mathrm{P}=0.815)$ between fat grafting sites and operation methods.

\section{Fat harvesting, refinement, and injection}

The fat donor sites were the abdomen and flank for 91 patients and the thigh in 11 patients. Fat was harvested by Coleman's technique [7]. First, a tumescent solution of $1,000 \mathrm{~mL}$ normal saline, $20 \mathrm{~mL} 2 \%$ lidocaine, and $1 \mathrm{~mL}$ 1:100,000 epinephrine was injected into the fat harvest area. We then waited for approximately 20 minutes to allow for dispersion of the tumescent solution. Using a two-hole Coleman harvesting cannula (Tulip Medical Products, San Diego, CA, USA) and a $50 \mathrm{~mL}$ syringe, fat harvesting was performed. The fat graft was collected with a $10 \mathrm{~mL}$ Luer Lock syringe fitted directly on the cannula, and then transferred to a standard centrifuge. After centrifugation at $3,000 \mathrm{rpm}$ for 3 minutes, the supernatant oil was wicked off and the fluid at the dependent portion of the syringe was decanted. The fat graft was placed into syringes for transfer through Coleman cannulas into the soft-tissue deformities. Small aliquots of fat were injected through multiple passes and tissue planes to improve the likelihood of graft implantation, overcorrecting the defects according to the surgeon's judgment.

\section{Postoperative follow-up}

Before fat grafting $\left(\mathrm{T}_{0}\right)$, the breast was evaluated by physical examination, gross photography, and ultrasonography at our outpatient clinic. We repeated these evaluations within 1 month after fat injection $\left(T_{1}\right)$. Between 1 month and 6 months $\left(T_{2}\right)$ after fat grafting, the breast was evaluated by physical examination alone. At 6 months after fat grafting $\left(T_{3}\right)$, we again evaluated the breast by physical examination, gross photography, and ultrasonography. The evaluations at $T_{2}$ and $T_{3}$ were used to assess the presence of complications.

\section{Fat reabsorption rate}

For 38 patients who underwent upper portion fat injection, fat reabsorption rate was calculated using ultrasonography measurements of the depth of fat tissue from skin to pectoralis muscle fascia. The fat thickness was measured at the middle points of the lines between the nipple (if the patient has no nipple, the maximal projection of breast mound substituted the point of nipple) and the points of the outer edge of the gland at the 12-o'clock position (Fig. 1). The depth of fat tissue was measured at the same area at times T0, T1, and T3. The reabsorption rate was calculated using the following formula, with $\mathrm{A}, \mathrm{B}$, and $C$ representing the measurements at $T_{0}, T_{1}$, and $T_{3}$, respectively: $[1-(\mathrm{C}-\mathrm{A}) /(\mathrm{B}-\mathrm{A})] \times 100)($ Fig. 2). 


\section{Locoregional recurrence}

After mastectomy, regular follow-up was performed with ultrasonography and physical examination by an oncologic surgeon to detect loco-regional recurrence in the fat injection group. Regular follow-up was performed in a similar manner in a group of control patients who were not injected autologous fat. Other methods, such as abdominal ultrasonography, whole body bone scan, and abdomino-pelvic and chest computed tomography, were used to detect distant metastasis.

\section{Fig. 1. The point of measurement of the fat depth by} ultrasonography

The fat thickness was measured at the point as shown by the asterisks, namely, the middle points of the lines between the nipple and the points of the outer edge of the gland at 12-o'clock position.
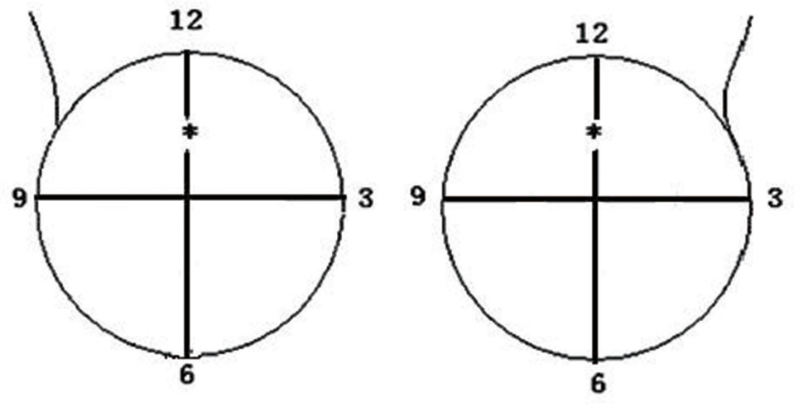

\section{RESULTS}

During the initial injection, the average volume of injection was $49.3 \mathrm{~mL}$ (range, 10-183 mL). Fat injection was performed only once for the majority of patients $(n=94)$. For the majority of patients, the location of injection was the upper pole of the breast, followed by axilla, lower pole and medial portion (Table 1).

Fat graft was repeated in 29 of the 102 patients (28.4\%). Of these 29,25 patients underwent fat graft a total of two times, and 4 patients underwent fat graft a total of three times. The mean volume of the additional injections was $37 \mathrm{~mL}$ (range, 24-80 mL). At the initial procedure, the volume of fat injected in those patients who underwent repeat injections was slightly lower than the volume injected in the patients who did not have a repeat injection $(42 \mathrm{~mL} v s .50 \mathrm{~mL})$.

\section{Complications}

Complications, including fat necrosis and cyst formation, detected by ultrasonograpy of the fat injection site, occurred in 18 of the 102 patients (17.6\%) (Table 2, Fig. 3). No infection or implant rupture was noted. Details regarding each complication are provided in Table 2 . The presence of a complication was associated with the volume of fat injected. The mean total volume of fat injected into the breasts was $67.5 \mathrm{~mL}$ for those patients who developed complications following the procedure, whereas

Fig. 2. Measurement of fat reabsorption rate by ultrasonography

(A) A: thickness before fat graft. (B) B: thickness within 1 month after fat graft. (C) C: thickness at more than 6 months after fat graft. The thickness was measured from skin to pectoralis muscle fascia, $C$ fat reabsorption rate was calculated by $[1-(C-A) /(B-A)] \times 100$.
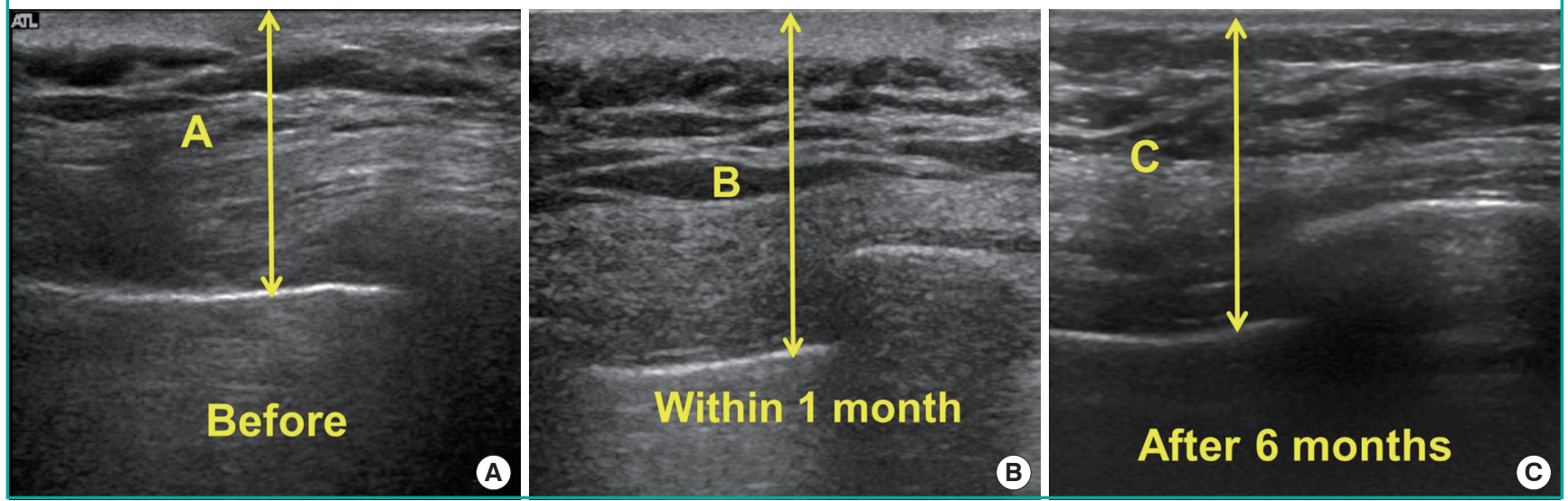

Table 1. Location of fat graft

\begin{tabular}{|lcccc|}
\hline Type of reconstruction & Upper (\%) & Medial (\%) & Lower (\%) & Axilla (\%) \\
\hline Transverse rectus abdominis myocutaneous & 57.1 & 4.8 & 28.6 & 9.5 \\
Latissimus dorsi & 60.7 & 16.1 & 10.7 & 12.5 \\
Implant & 67.7 & 7.7 & 7.7 & 17.0 \\
Total & 63.4 & 10.6 & 12.0 & 14.1 \\
\hline
\end{tabular}

There was no significant difference between fat injection site and type of reconstruction $(P=0.815)$. 
Table 2. Complications following fat graft

\begin{tabular}{|c|c|c|c|c|c|c|}
\hline Patient & Complication & Age (yr) & Reconstruction method & Adjuvant therapy & Number of graft & Graft volume $(\mathrm{mL})^{\mathrm{a})}$ \\
\hline 1 & Cystic lesion & 43 & Implant & None & 1 & 49 \\
\hline 2 & Cystic lesion & 52 & TRAM & CTx & 2 & 101 \\
\hline 3 & Fat necrosis & 34 & LD & CTx & 1 & 45 \\
\hline 4 & Cystic lesion & 43 & LD & None & 1 & 82 \\
\hline 5 & Fat necrosis & 52 & TRAM & RTx & 1 & 20 \\
\hline 6 & Fat necrosis & 47 & Implant & CTx & 1 & 20 \\
\hline 7 & Cystic lesion & 51 & TRAM & None & 1 & 93 \\
\hline 8 & Cystic lesion & 38 & LD & CTx, RTx & 2 & 68 \\
\hline 9 & Fat necrosis & 52 & Implant & None & 1 & 66 \\
\hline 10 & Fat necrosis & 60 & LD & None & 1 & 150 \\
\hline 11 & Cystic lesion & 35 & LD & None & 1 & 55 \\
\hline 12 & Fat necrosis & 43 & $L D$ & None & 1 & 60 \\
\hline 13 & Cystic lesion & 36 & LD & None & 1 & 55 \\
\hline 14 & Fat necrosis & 38 & LD & CTx & 2 & 114 \\
\hline 15 & Fat necrosis & 57 & LD & None & 1 & 183 \\
\hline 16 & Cystic lesion & 37 & Implant & None & 2 & 205 \\
\hline 17 & Fat necrosis & 58 & Implant & CTx & 1 & 40 \\
\hline 18 & Fat necrosis & 65 & LD & None & 1 & 80 \\
\hline
\end{tabular}

\section{Fig. 3. Complication after the fat graft}

(A) A 60-year-old woman, who initially underwent latissimus dorsi myocutaneous flap reconstruction, was noted to have fat necrosis at 38 months after fat graft with $150 \mathrm{~mL}$ in the left breast. (B) A 43-year-old woman, who initially underwent implant reconstruction, was noted to have a cyst 13 months after fat graft with $49 \mathrm{~mL}$ in the left breast.
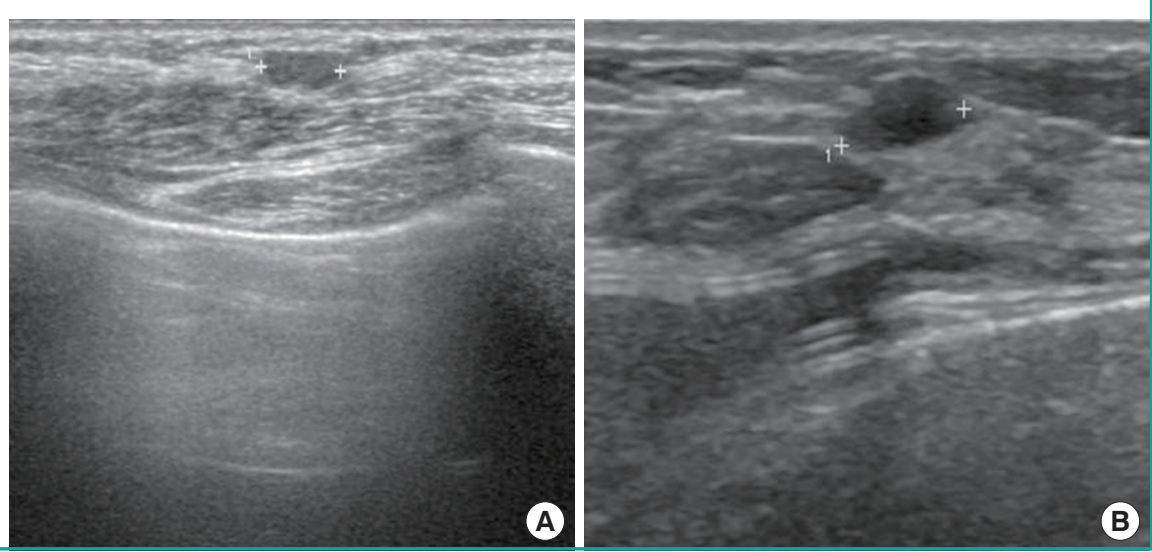

the fat volume was $45.2 \mathrm{~mL}$ for those without complications. Of the 18 patients with complications, 10 had fat necrosis, and 8 had cystic lesions. Among them, only three patients complained of a palpable mass, which led to a biopsy; pathologic examination confirmed as fat necrosis. All cases complained palpable mass or not was conservatively managed with no other procedure like surgical excision or drainage. But regular follow up was done.

\section{Fat reabsorption rate and loco-regional recurrence}

For the 38 patients in whom the fat reabsorption rate was calculated, the mean rate was $32.9 \%$ (range, 25\%-52\%) (Fig. 4). At before and after 6 months of fat injection, the gross photograph were shown in patient underwent implant reconstruction and
Fig. 4. The thickness measured by ultrasonography

Measurements of 38 patients who underwent upper pole fat injection from skin to pectoralis muscle fascia.

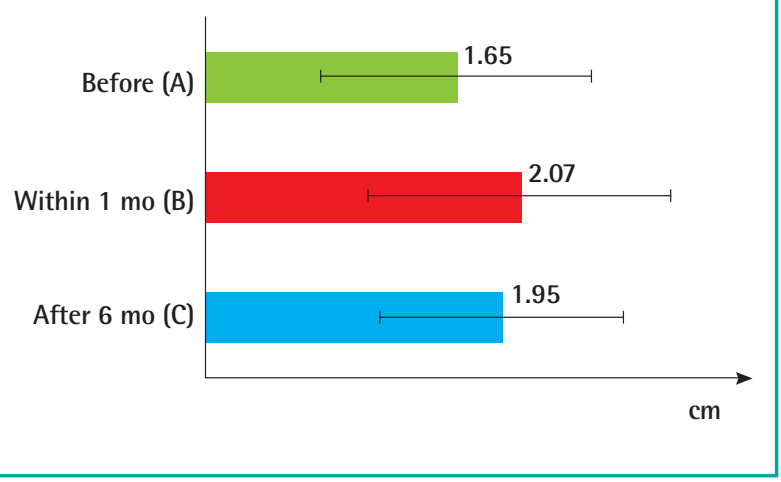




\section{Fig. 5. A case of fat graft}

(A, B) Preoperative views of a 34-year-old patient with left breast traction rippling caused by a textured implant and scant upper pole volume. (C, D) Results at 9 months postoperatively, following a single injection of $40 \mathrm{~mL}$ of autologous fat to the left breast upper pole.
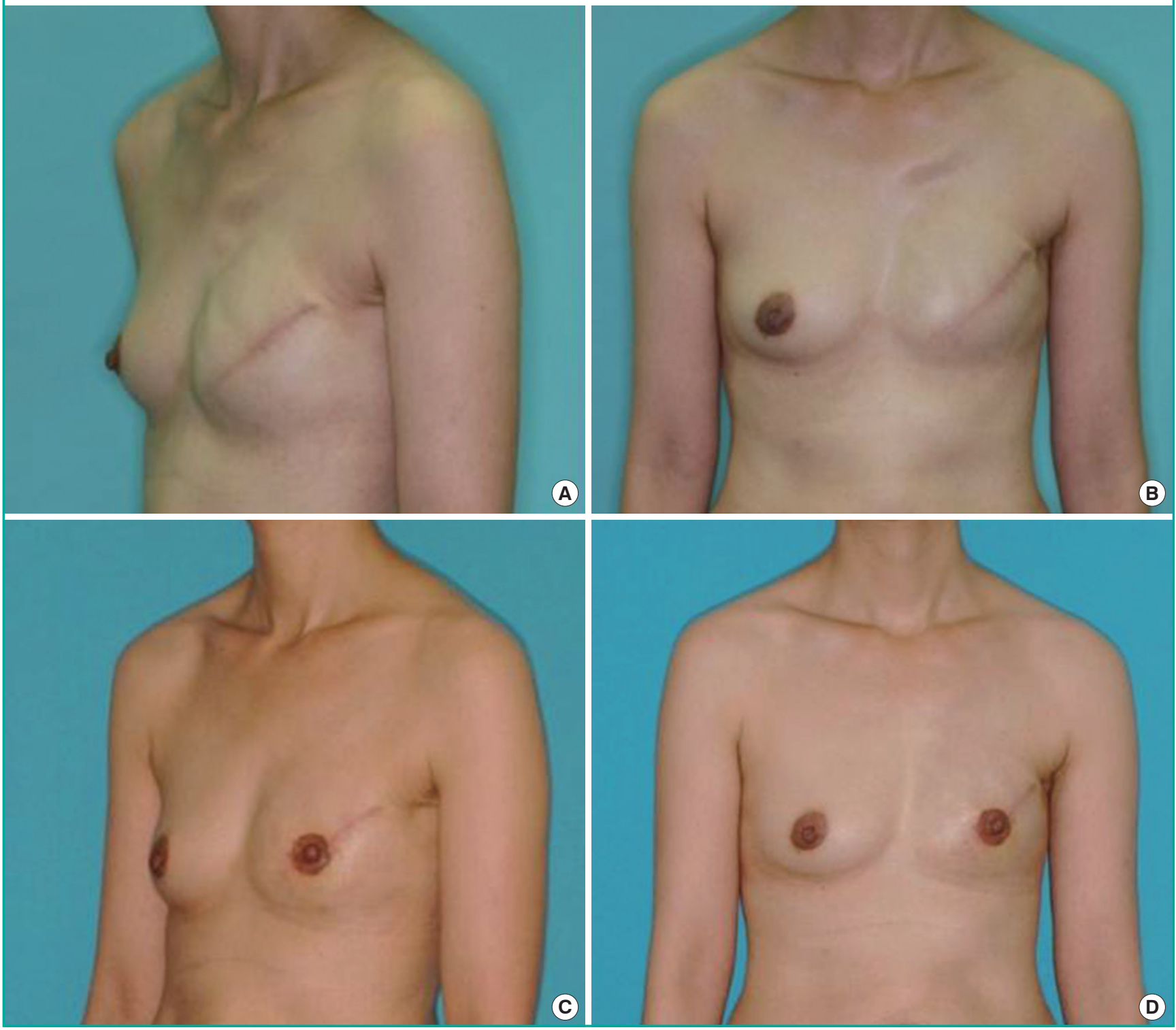

\section{LD myocutaneous flap reconstruction (Figs. 5, 6).}

Loco-regional cancer recurrence was noted in 1 patient $(0.9 \%)$ in the fat injection group. In a control group who did not undergo fat graft, 9 of 449 patients (2.0\%) were noted to have a recurrence in the same duration of follow-up. There was no significant difference between fat graft group and control group.

\section{DISCUSSION}

Contour deformity and irregularity of the reconstructed breast can be a challenging problem. As the various methods of primary breast reconstruction have improved, expectations of patients and surgeons for a natural appearing, aesthetically appealing, and well-contoured final result have been raised. The main goal of breast reconstruction is to produce a natural appearing and symmetric mound of breast. However, in the immediate or later postoperative period, the contour of the reconstructed breast may become unsatisfactory, and further correction of the breast shape and contour at the time of other secondary procedures, such as nipple reconstruction, is not uncommon [1].

In our study, fat graft was performed in all quadrants of the breast, as well as the axilla. The most frequent fat graft area was the upper portion regardless of the reconstruction procedure. For LD myocutaneous flap reconstruction, a lack of volume oc- 


\section{Fig. 6. A case of fat graft}

$(A, B)$ Preoperative views of a 42-year-old patient with an immediate right breast latissimus dorsi myocutaneous island transposition flap. (C, D) Results at 6 months postoperatively, following a single injection of $60 \mathrm{~mL}$ autologous fat to the right upper pole.
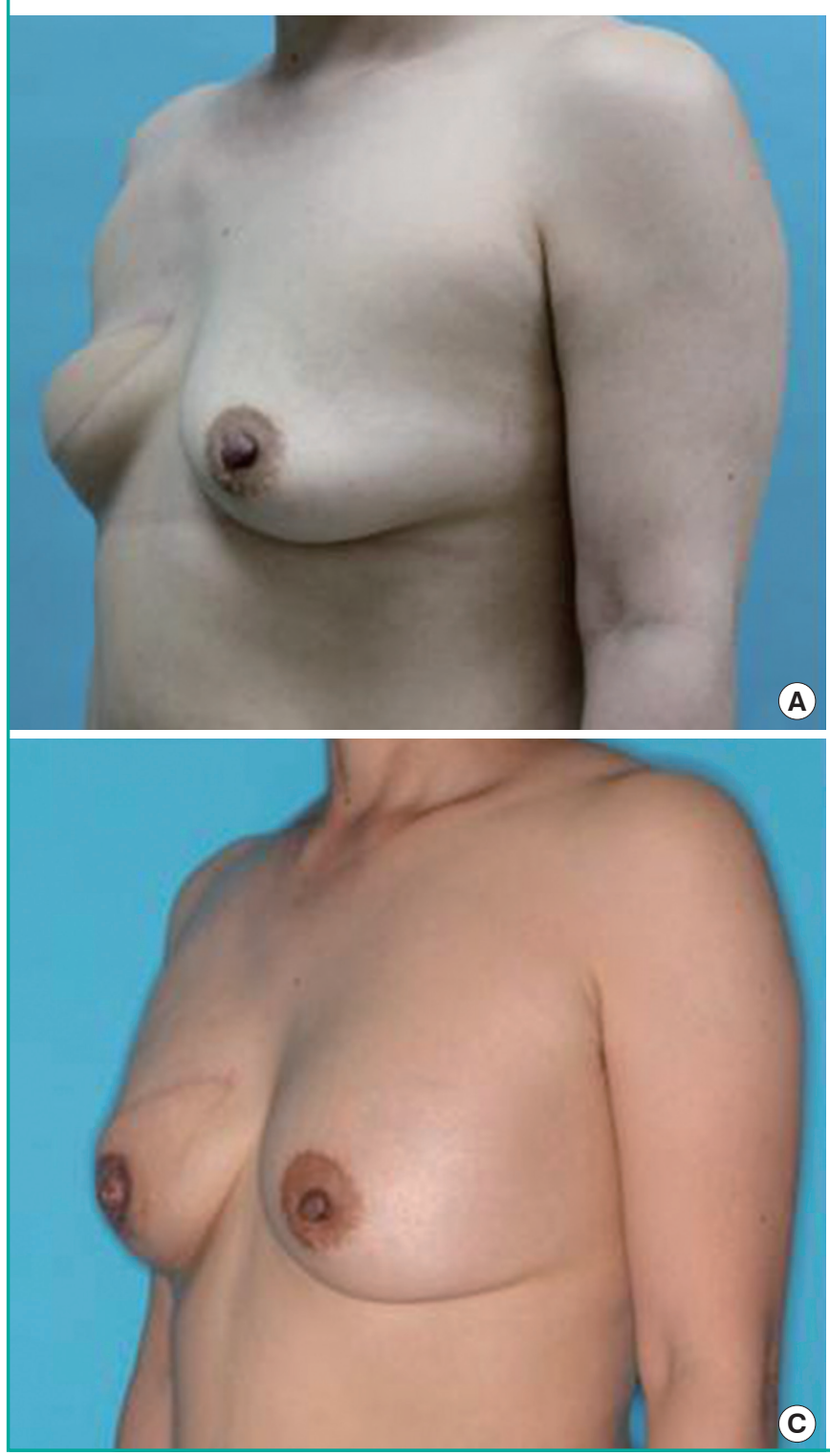

C)
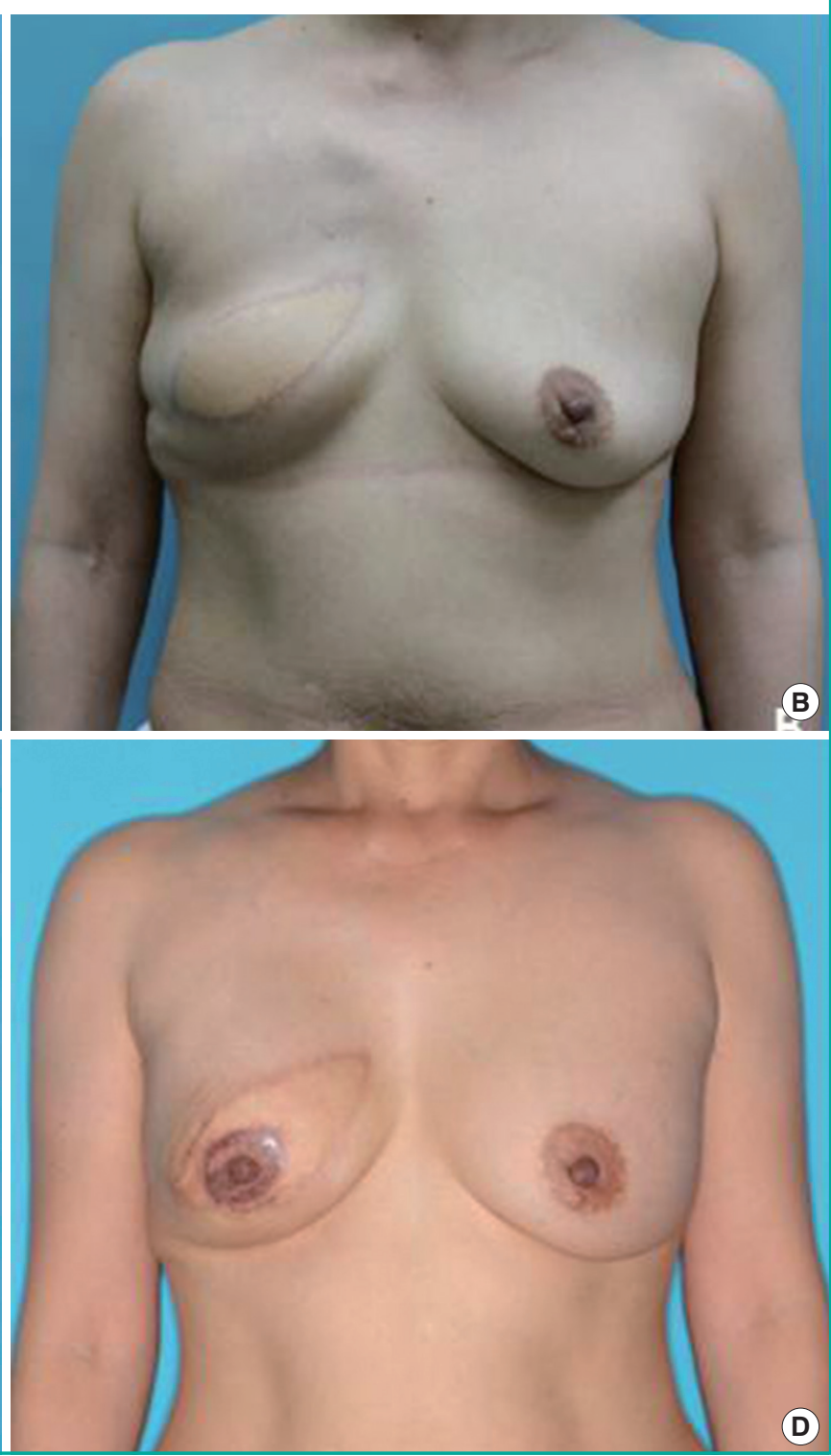

curred in many of our cases. When constructing a breast mound with a small volume, a relative lack of upper quadrant fullness is not unexpected. For implant reconstruction, it is difficult to obtain a natural breast shape with a round implant, although use of an anatomically-shaped implant improves the appearance. Those cases could be indications for secondary refinement with fat graft.

The safety of microfat graft is evident in our series, as indicated by our low complication rate of $17.6 \%$, which was limited to only minor complications. This rate is compatible with, although slightly higher than, rates reported in the literature. In 2005 , Spear et al. [3] reported an $8.5 \%$ complication rate for the correction of contour deformities in 37 patients, and in 2011, Losken et al. [1] reported an $11 \%$ complication rate in 107 patients. One potential reason for our higher rate was our use of a higher fat graft volume. Minimizing the volume grafted with each pass of the cannula will maximize the surface area of contact between the grafted fat and the recipient tissue. The proximity of the newly grafted fat to a blood supply encourages survival and minimizes the potential for fat necrosis and later calcification. Thus, it is not surprising that with a larger volume grafted, there was less surface contact between the grafted fat and the recipient tissue. The larger mean graft volume in our group with complications compared to those without complica- 
tions $(67.5 \mathrm{~mL}$ vs. $45.2 \mathrm{~mL})$ further illustrates the importance of graft volume. Another potential reason for the disc between our complication rate and those of previous studies was our use of a larger syringe with a large-caliber cannula for fat harvesting. Coleman and Saboeiro [7] recommended the use of a $10 \mathrm{~mL}$ syringe for fat harvesting to reduce the pressure generated during the harvesting procedure and thereby potentially preserve the fat parcels [8]. We used a $50 \mathrm{~mL}$ syringe with a $3 \mathrm{~mm}$ caliber cannula to lead high negative pressure and to derive large fat parcels, which may have damaged the fat parcels and negatively influenced fat graft outcomes.

The fat reabsorption rate in this study was 32.9\% (range, 25\%$52 \%$ ). Clinically, volume loss between $40 \%$ and $60 \%$ has been reported after fat grafting, which usually occurs after 4 to 6 months $[9,10]$. Although over-correction is performed by some surgeons, the need for re-grafting is often required and should be discussed with the patient initially. In our study, $28.4 \%$ of patients underwent more than one fat grafting procedure to achieve the optimal cosmetic result. In Delay's [9] review of 200 breast reconstructions, $22 \%$ underwent multiple fat grafts. Missana et al. [4] demonstrated a somewhat lower repeat injection rate of $15 \%$. There are many methods for fat refinement, including filtration, cotton gauze rolling, and centrifugation. Centrifugation is the most commonly used method, and we centrifuged the harvested material at 3,000 rpm for 3 minutes, as this allowed us to obtain a higher adipocyte concentration. Fisher et al. [11] found that among the fat refinement techniques, the cotton rolling method produced the highest stromal vascular fraction cell count; he recommended using this method when the volume of the graft was small. However, this method becomes more cumbersome for larger volume injections. The LipiVage System like Harvest-jet is also relatively easy to use and has been shown to yield a sufficient number of viable adipocytes [12].

In 1987, the American Society of Plastic Surgeons prohibited the use of autologous fat grafting to the female breast because of concerns that it would interfere with subsequent cancer screening and that adipocytes may have tumor-promoting effects [4]. However, in terms of cancer screening, Pierrefeu-Lagrange et al. [13] reported the use of multimodal radiographic imaging in 30 patients who underwent fat graft to the reconstructed breast, and it was felt that fat grafting did not interfere with oncologic surveillance in these patients. In our current study, the cancer locoregional recurrence rate was $0.9 \%$; that is, it is proposed that no tumors recurred due to fat graft compared with control group recurrence $(2.0 \%)$. Our cancer recurrence rate after fat grafting was significant lower than the rates reported by other studies. Rigotti et al. [14] reported the overall incidence of lo- coregional or distant recurrence to be 5.9\% after a 5-year followup period. Previous studies have explored the potential tumorigenic effects of fat cells. Ando and Catalano [15] reported that leptin is a cytokine-like protein secreted from adipocytes that plays a role in cell proliferation and neovascularization in malignant and nonmalignant human cellular lines. Pearl et al. [6] suggested that transference of adipose tissue and adipose-derived stem cells to an environment that has been in the locale of previous malignant change may constitute an unacceptable risk. Further studies are necessary to more fully define the roles of adipocytes, preadipocytes, and adipose-derived stem cell in tumorigenesis, recurrence, and metastasis.

One of our patients who showed locoregional recurrence originally had a history of partial mastectomy and axillary lymph node dissection under diagnosis of invasive ductal carcinoma, and 20 years later, recognized a mass-like lesion on the same breast which led her to receive total mastectomy under the same pathologic diagnosis. The patient underwent an implant-based breast mound reconstruction afterwards, and a nipple-areolar complex reconstruction surgery followed 9 months later together with $40 \mathrm{~mL}$ of fat graft on depressed upper pole area. Twenty three months from the fat grafting procedure, a regular followup whole body bone scan revealed increased uptake in the sternum suspicious of bone metastasis. A subsequent positron emission tomography-computed tomography scan further confirmed multiple sites of mestastasis in the sternum, left scapula, vertebral bodies, left pubic bone, and possible lung metastasis in the left lung base. The patient currently is under chemotherapy showing stable state of the disease, and additional chemotherapy will be provided.

Although our results are encouraging, we recognize that this study has limitations. To provide definitive guidance to surgeons undertaking fat graft for breast reconstruction deformities, larger prospective studies are required. In particular, investigations to determine the optimal volume of fat graft and the number of grafts to use would be of value. We measured the thickness by ultrasonography in determining the fat reabsorption rate, but several technologies, including 3-dimensional cameras, can be used to create three-dimensional images of the breast, which permit accurate evaluation of size, volume, and contour [16].

In this study of fat graft as a tool for acquired contour deformities of the reconstructed breast, we have demonstrated efficient outcomes and acceptable complication rate. It suggests that fat graft represents a promising intervention for the management of a common and distressing problem for patients who have undergone postmastectomy breast reconstruction. However, several issues, such as cancer recurrence, postoperative surveillance, 
fat reabsorption rate, and the role of stromal vascular fraction cells, should continue to be explored to maximize the safety and outcomes of autologous fat grafting to the breast.

\section{REFERENCES}

1. Losken A, Pinell XA, Sikoro K, et al. Autologous fat grafting in secondary breast reconstruction. Ann Plast Surg 2011;66: 518-22.

2. Report on autologous fat transplantation. ASPRS Ad-Hoc Committee on New Procedures, September 30, 1987. Plast Surg Nurs 1987;7:140-1.

3. Spear SL, Wilson HB, Lockwood MD. Fat injection to correct contour deformities in the reconstructed breast. Plast Reconstr Surg 2005;116:1300-5.

4. Missana MC, Laurent I, Barreau L, et al. Autologous fat transfer in reconstructive breast surgery: indications, technique and results. Eur J Surg Oncol 2007;33:685-90.

5. Gutowski KA; ASPS Fat Graft Task Force. Current applications and safety of autologous fat grafts: a report of the ASPS fat graft task force. Plast Reconstr Surg 2009;124:272-80.

6. Pearl RA, Leedham SJ, Pacifico MD. The safety of autologous fat transfer in breast cancer: lessons from stem cell biology.J Plast Reconstr Aesthet Surg 2012;65:283-8.

7. Coleman SR, Saboeiro AP. Fat grafting to the breast revisited: safety and efficacy. Plast Reconstr Surg 2007;119:77585 .
8. Toledo LS. Syringe liposculpture. Clin Plast Surg 1996;23: 683-93.

9. Delay E. Lipomodeling of the reconstructed breast. In: Spear SL, editor. Surgery of the breast: principles and art. Philadelphia: Lippincott Williams \& Wilkins; 2006. p.912-33.

10. Horl HW, Feller AM, Biemer E. Technique for liposuction fat reimplantation and long-term volume evaluation by magnetic resonance imaging. Ann Plast Surg 1991;26:248-58.

11. Fisher C, Grahovac TL, Schafer ME, et al. Comparison of harvest and processing techniques for fat grafting and adipose stem cell isolation. Plast Reconstr Surg 2013;132:351-61.

12. Ferguson RE, Cui X, Fink BF, et al. The viability of autologous fat grafts harvested with the LipiVage system: a comparative study. Ann Plast Surg 2008;60:594-7.

13. Pierrefeu-Lagrange AC, Delay E, Guerin N, et al. Radiological evaluation of breasts reconstructed with lipomodeling. Ann Chir Plast Esthet 2006;51:18-28.

14. Rigotti G, Marchi A, Galie M, et al. Clinical treatment of radiotherapy tissue damage by lipoaspirate transplant: a healing process mediated by adipose-derived adult stem cells. Plast Reconstr Surg 2007;119:1409-22.

15. Ando $S$, Catalano $S$. The multifactorial role of leptin in driving the breast cancer microenvironment. Nat Rev Endocrinol 2012;8:263-75.

16. Choi M, Small K, Levovitz C, et al. The volumetric analysis of fat graft survival in breast reconstruction. Plast Reconstr Surg 2013;131:185-91. 